\title{
Differences between chronological and brain age are related to education and self-reported physical activity
}

\author{
Jason Steffener ${ }^{\mathrm{a}, \mathrm{b}, \mathrm{c}, *}$, Christian Habeck ${ }^{\mathrm{d}}$, Deirdre O'Shea ${ }^{\mathrm{d}, \mathrm{e}}$, Qolamreza Razlighi ${ }^{\mathrm{d}}$, \\ Louis Bherer ${ }^{\mathrm{a}, \mathrm{b}, \mathrm{c}}$, Yaakov Stern ${ }^{\mathrm{d}}$ \\ a PERFORM Centre, Concordia University, Montreal, Québec, Canada \\ ${ }^{\mathrm{b}}$ Centre de Recherche de l'Institut de Gériatrie de Montréal, Montréal, Québec, Canada \\ ${ }^{\mathrm{c}}$ Department of Psychology, Concordia University, Montreal, Québec, Canada \\ ${ }^{\mathrm{d}}$ Cognitive Neuroscience Division, Department of Neurology and Taub Institute for Research on Alzheimer's Disease and the Aging Brain, Columbia \\ University College of Physicians and Surgeons, New York, NY, USA \\ ${ }^{\mathrm{e}}$ Department of Clinical and Health Psychology, University of Florida, Gainesville, FL, USA
}

\section{A R T I C L E I N F O}

\section{Article history:}

Received 14 May 2015

Received in revised form 13 January 2016

Accepted 25 January 2016

Available online 1 February 2016

\section{Keywords:}

Healthy aging

Lifestyle

Gray matter volume

Brain age

Education

Physical activity

\begin{abstract}
A B S T R A C T
This study investigated the relationship between education and physical activity and the difference between a physiological prediction of age and chronological age (CA). Cortical and subcortical gray matter regional volumes were calculated from 331 healthy adults (range: 19-79 years). Multivariate analyses identified a covariance pattern of brain volumes best predicting $C A\left(R^{2}=47 \%\right)$. Individual expression of this brain pattern served as a physiologic measure of brain age (BA). The difference between CA and BA was predicted by education and self-report measures of physical activity. Education and the daily number of flights of stairs climbed (FOSC) were the only 2 significant predictors of decreased BA. Effect sizes demonstrated that BA decreased by 0.95 years for each year of education and by 0.58 years for 1 additional FOSC daily. Effects of education and FOSC on regional brain volume were largely driven by temporal and subcortical volumes. These results demonstrate that higher levels of education and daily FOSC are related to larger brain volume than predicted by CA which supports the utility of regional gray matter volume as a biomarker of healthy brain aging.
\end{abstract}

(c) 2016 Elsevier Inc. All rights reserved.

\section{Introduction}

Gray matter volume decline is a highly visible aspect of the chronological aging process resulting from neural shrinkage and neuronal loss (Terry et al., 1987). These neural changes are detectable with magnetic resonance imaging (MRI) as volumetric declines in subcortical regions and throughout the cortical mantel (Dale et al., 1999; Sowell et al., 2004). Although volumetric decline is a common aspect of aging, the rate and degree of decline is highly variable across regions of the brain and between individuals (Raz et al., 2010). Furthermore, differences in lifetime exposures, such as years of education or physical activity, have been associated with differential amounts of gray matter volumetric decline with advancing age (Ahlskog et al., 2011; Erickson et al., 2010; Nithianantharajah and Hannan, 2009).

Interindividual variability in genetics and development along with positive and negative effects of lifetime exposure will result in

\footnotetext{
* Corresponding author at: Department of Psychology, Concordia University, 7141 Sherbrooke Street West, PC 2.302, Montreal, QC H4B 1R6, Canada. Tel.: 514-8482424 \#4453; fax: 514-848-4513.

E-mail address: jason.steffener@concordia.ca (J. Steffener).
}

different quantities of brain volume loss. Several investigators have suggested the concept of physiological brain age (BA) where the difference between chronological age (CA) and predicted age, based on brain measures, serves as a more informative marker of brain health than CA alone (Franke et al., 2010; Irimia et al., 2014). Regional brain volume measures would be useful for calculating a physiological BA measurement.

In this study, we used regional measures of gray matter volume from 331 healthy adults across the life span to derive a biomarker of BA. We defined the difference between CA and BA as a marker of whether the brain is younger or older than expected. We then investigated whether this difference was related to lifetime exposures including years of education and self-reported assessments of physical activity. Such relationships would suggest that certain lifetime exposures help maintain the brain in a more "youthful" state.

\section{Materials and methods}

\subsection{Participants}

Data from 331 healthy adults between the ages of 19 and 79 were included in this study. Participants were drawn from 3 
different studies from our laboratory using the same testing apparatus, procedures, and MRI. Table 1 lists the number of participants by decade, sex, and study. Participants were recruited using market-mailing procedures from within 10 miles of our northern Manhattan, NY, USA site to equalize the recruitment approaches across the life span. Participants who responded to the mailing were telephone screened to ensure that they met basic inclusion criteria (right handed, English speaking, no psychiatric or neurologic disorders, normal, or corrected-to-normal vision). All participants found eligible via the initial telephone screen were further screened in person with structured medical, neurologic, psychiatric, and neuropsychological evaluations to ensure that they had no neurologic or psychiatric disease or cognitive impairment. The screening procedure included a detailed interview that excluded individuals with a self-reported history of major or unstable medical illness, significant neurologic history (e.g., epilepsy, brain tumor, stroke), history of head trauma with loss of consciousness for $>5$ minutes or history of Axis I psychiatric disorder (Association, A. P, 1994). Individuals taking psychotropic medications were also excluded. Global cognitive functioning was assessed with the Mattis Dementia Rating Scale, on which a score of at least 133 was required for retention in the study (Mattis, 1988). This study was approved by the Internal Review Board of the College of Physicians and Surgeons of Columbia University, and written informed consent was obtained from all participants prior to study participation and after the purpose and risks of the study were explained. Participants were compensated for their participation in the study.

\subsection{Measures of lifetime exposures}

Lifetime exposures included measures of education and physical activity. Education was assessed as the number of years engaged in formal education. Physical activity was assessed using a questionnaire containing 9 questions about the amount of time spent doing various physical activities. These activities included walking/hiking, jogging, running, bicycling, aerobic exercise, lap swimming, tennis/ squash/racquetball, low intensity exercise, and flights of stairs climbed daily (FOSC). The FOSC daily was coded as follows: none, $1-2,3-4,5-9,10-14$, and $>15$. These values were coded as $0,1.5$, $3.5,7,12$, and 16 . The other questions referred to the amount of time spent within 1 week and were coded as follows: none, 1-19 minutes, 20-50 minutes, 1 hour, 1.5 hours, $2-3$ hours, 4-6 hours, and $>7$ hours. This questionnaire is similar to that used in other assessments of physical activity (Chao et al., 2004; Thacker et al., 2008). For all physical activities, these measures were converted to metabolic equivalent of task (MET) (Ainsworth et al., 2000; Bassett et al., 1997) and a total MET score was calculated.

\subsection{Image acquisition procedure}

MRI images were acquired in a 3.0T Philips Achieva Magnet using a standard quadrature head coil. A T1-weighted scout image

Table 1

Sample sizes split by decade, sex, and study

\begin{tabular}{|c|c|c|c|c|c|}
\hline Age group & $\begin{array}{l}\text { Total N } \\
\text { F/M }\end{array}$ & $\begin{array}{l}\text { Study } 1 \mathrm{~N} \\
\mathrm{~F} / \mathrm{M}\end{array}$ & $\begin{array}{l}\text { Study } 2 \text { N } \\
\text { F/M }\end{array}$ & $\begin{array}{l}\text { Study } 2 \& 3 \mathrm{~N} \\
\text { F/M }\end{array}$ & $\begin{array}{l}\text { Study } 3 \mathrm{~N} \\
\text { F/M }\end{array}$ \\
\hline$>30$ & $43 / 21$ & $9 / 4$ & $10 / 5$ & $10 / 3$ & $14 / 9$ \\
\hline $30-39$ & $32 / 19$ & $3 / 1$ & $2 / 1$ & $4 / 1$ & $23 / 16$ \\
\hline $40-49$ & $14 / 19$ & $0 / 0$ & $0 / 0$ & $0 / 0$ & $14 / 19$ \\
\hline $50-59$ & $20 / 23$ & $0 / 0$ & $1 / 0$ & $0 / 1$ & $19 / 22$ \\
\hline $60-69$ & $46 / 45$ & $7 / 9$ & $16 / 10$ & $15 / 19$ & $8 / 7$ \\
\hline 70 and older & $27 / 22$ & $1 / 0$ & $4 / 2$ & $2 / 2$ & $20 / 18$ \\
\hline Total & $182 / 149$ & $20 / 14$ & $33 / 18$ & $31 / 26$ & $98 / 91$ \\
\hline
\end{tabular}

was acquired to determine the participant's position. One hundred sixty-five contiguous 1-mm coronal T1-weighted images of the whole brain were acquired for each participant with an Magnetization Prepared Rapid Acquisition Gradient Echo sequence using the following parameters: TR $6.5 \mathrm{~ms}$, TE $3 \mathrm{~ms}$; flip angle $8^{\circ}$, acquisition matrix $256 \times 256$, and $240 \mathrm{~mm}$ field of view. A neuroradiologist reviewed the anatomic scans to identify any potentially clinically significant findings.

\subsection{FreeSurfer methods}

Each participant's structural T1 scans were reconstructed using FreeSurfer (Fischl, 2012) (http://surfer.nmr.mgh.harvard.edu/). The accuracy of Freesurfer's subcortical segmentation and cortical parcellation (Fischl et al., 2002, 2004) has been reported to be comparable with manual labeling. Each participant's white and gray matter boundaries as well as gray matter and cerebrospinal fluid boundaries were visually inspected slice by slice by an experienced user, manual control points were added in the case of any visible discrepancy, and reconstruction was repeated until we reached satisfactory results within every participant. The subcortical structure borders were plotted by FreeView visualization tools and compared against the actual brain regions. In case of discrepancy, they were corrected manually. In total, this procedure quantified 84 regions, 16 subcortical and 68 cortical.

\subsection{Scaled subprofile modeling}

We used the scaled subprofile modeling (Moeller et al., 1987; Spetsieris and Eidelberg, 2010) approach to calculate physiological BA based on regional gray matter volumes from cortical and subcortical locations using the principal components analysis toolbox (http://groups.google.-com/group/gcva) (Habeck and Stern, 2007; Habeck et al., 2005). Briefly, the 84 gray matter volume regions of interest were subjected to a principal component analysis. This produced a series of principal component images, $v_{i}$, and their respective subject scaling factors $\left(\mathrm{SSF}_{\mathrm{i}}\right)$, which are each individual's expression of that respective principal component. The number of components comprising approximately $95 \%$ of the variance of the structural data was retained. Predicted Age (pAge) was calculated by regressing the SSF's taken from this subset of principal components against CA controlling for total intracranial volume, study (which of the studies they were recruited for), and sex:

$$
\begin{aligned}
\text { pAge }= & \beta_{0}+\operatorname{SSF}_{1} \beta_{1}+\operatorname{SSF}_{2} \beta_{2}+S_{S F_{3}} \beta_{3}+\ldots+\beta_{x} T I V+\beta_{y} \text { Study } \\
& +\beta_{z} \operatorname{Sex}+\varepsilon
\end{aligned}
$$

Both sex and study were dummy-coded, with female and study 1 as the references. The weights from the SSF variables were used to combine the respective eigenimages, $v_{i}$, to produce an age-related brain covariance pattern. This was then projected back into the original data to calculate the expression of this pattern for each participant. This value served as the BA measure. The stability of the regions within the resultant pattern was assessed using 1000 bootstrap resamples and tested with bias-corrected, accelerated confidence intervals.

For each individual, chronological age and brain age (CA-BA) indicated whether their BA was older or younger than their CA. Positive values indicate their BA was younger than their CA would predict, whereas a negative value indicates the BA was older than their CA. We then tested whether the measures of education and physical activity were related to this difference.

Similar to assessing regional contributions to the BA map, regional contributions to the CA-BA relationship with education 
and physical activity were also calculated. The stability of the regions was assessed using 1000 bootstrap resamples and tested with bias-corrected, accelerated confidence intervals. Regions were identified as being significantly related to the lifetime exposure measures if they had a $\mathrm{Z}$ score magnitude $>2$ and had effect sizes $>1$ of 84 of the total effect. An effect size of 1 of 84 of the total effect for each brain region signifies that each brain region is contributing equally to the overall covariance pattern and there is no regional concentration of the effect.

\section{Results}

\subsection{Global results}

The first 38 principal components comprised 95\% of the total variance in the regional gray matter volume structural data. In total, $64 \%$ of CA-related variance was accounted for, $47 \%$ by the individual expression of the covariance pattern and an additional $17 \%$ by the nuisance regressors of total intracranial volume, study, and sex. Statistics for the nuisance regressors were as follows: total intracranial volume $(B=58.7, t(324)=5.52, p<0.001)$, sex $(B=9.81$, $t(324)=6.89, p<0.001)$, and study $\left(R^{2}=0.007, F(3,324)=2.10, p=\right.$ $0.10)$. Study was dummy-coded according to which study the participant was part of or whether they were part of 2 studies; Table 1. A scatter plot of CA versus BA is shown in Fig. 1.

Education and MET scores for each of the 9 physical activities were regressed against the difference between CA-BA. The overall F-test was significant for this model $(\mathrm{F}(10,320)=2.14, p=0.021)$. CA-BA was significantly related to education $(B=0.95, t(320)=$ $2.84, p=0.0048)$ and $\operatorname{FOSC}(B=0.58, t(320)=2.84, p=0.0048)$. All other measures were nonsignificant as follows: walking/hiking $(B=0.041, t(320)=0.40, p=0.69)$, jogging $(B=0.16, t(320)=1.32$, $p=0.19)$, running $(B=-0.042, t(320)=-0.36, p=0.72)$, bicycling $(B=0.055, t(320)=0.56, p=0.57)$, aerobic exercise $(B=-0.063$, $t(320)=-0.82, p=0.41)$, lap swimming $(B=-0.072$, $t(320)=-0.55, p=0.58)$, tennis/squash/racquetball $(B=0.004$, $t(320)=0.027, p=0.98)$, and low intensity exercise $(B=-0.031$,

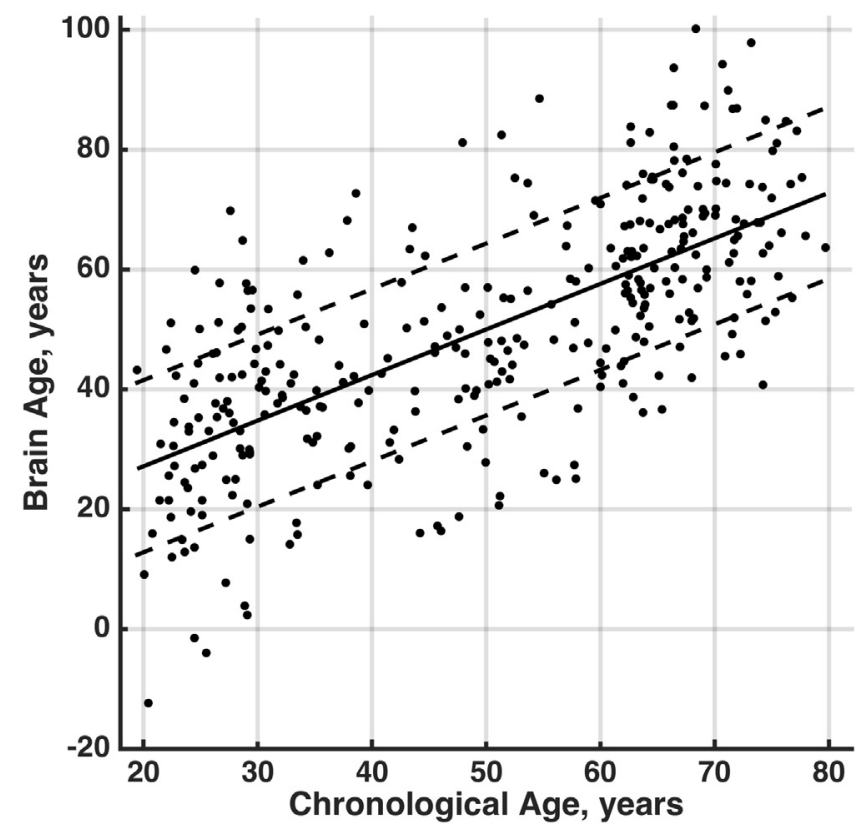

Fig. 1. Scatter plot of chronological age versus brain age in years after adjusting for nuisance variables. The solid line is the regression fit between the 2 variables, and the dashed lines are 1 standard deviation away from the regression fit. $t(320)=-0.13, p=0.90)$. The combined effect of 1 additional year of education combined with 1 additional flight of stairs climbed per day was related to a 1.53 years younger brain than CA would predict.

The nonsignificant regressors were removed and the regression analysis redone. The overall $F$-test was still significant for this model $(F(2,328)=9.45, p<0.001)$. CA-BA was significantly related to education $(B=0.99, t(328)=3.014, p=0.0028)$ and FOSC $(B=$ $0.60, t(328)=3.06, p=0.0024)$. The reduction in variance accounted for was not significant (difference in $R^{2}=0.0083, F(8$, $320)=0.36, p=0.94$ ).

The correlation table between the 9 physical activity measures and age is listed in Table 2. With increasing age, there is a decrease in the amount of time engaged in jogging, running, and aerobic exercise. Also included in Table 2 is the proportion of participants engaged in each activity after a median split (age 52) into young and old adults. This reiterates the age effect on the activities but it also demonstrates that walking and stair climbing are activities engaged in by the vast majority of participants regardless of age. The significant relationships between FOSC and CA-BA demonstrate a dose effect of stair climbing.

\subsection{Regional results}

Regional contributions to the CA-BA relationship with education and FOSC are listed in Table 3 and overlay in Fig. 2. For education, the effects included bilateral putamen, right amygdala, left lateral orbitofrontal gyrus, right superior frontal gyrus, right frontal pole, left precuneus, right precentral gyrus, bilateral superior temporal gyri, left middle temporal gyrus, left supramarginal gyrus, and right lateral occipital gyrus.

For FOSC, the effects were observed in the right amygdala, right accumbens, right rostral middle frontal gyrus, bilateral caudal anterior cingulate, right rostral anterior and posterior cingulate, left precuneus, right paracentral gyrus, right inferior and transverse temporal gyrus, left middle temporal gyrus, and the right insula.

\section{Discussion}

Brain aging can be conceptualized as comprising 2 mechanisms, the inevitable and universal effects of advancing age and the effects resulting from a lifetime of exposures. Lifetime exposures comprise both negative effects related to unhealthy lifestyle and injuries and positive effects resulting from a healthy lifestyle and enriched environments. The culmination of a lifetime of genetic, developmental, and lifetime exposures produces large variation in the physiological age of our brains. Having a physiological measurement of the age of the brain provides a means for assessing the effect of a lifetime of exposures on the brain. Not only does the CA-BA measure provide a more individualistic assessment, it could also provide a means of identifying exposures associated with maintenance of a more youthful brain in late life.

The current work used gray matter regional brain volumes as measures to compute a physiological age, (i.e., BA). This approach may be generalized to include other brain-based measures (e.g., white matter volumes, white matter hyperintensity burden, amyloid buildup) as well as non-brain-based measures such as bloodbased markers (e.g., complete blood count panels). Inclusion of additional measures would provide a more global estimation of physiological age and will likely account for a larger amount of the variance in CA.

The results of this study suggest that individual levels of education and FOSC have a positive effect on the brain. Increased values on either of these lifetime exposure measures were associated with significant increases in the CA-BA measure. Maintaining the 
Table 2

Correlations and group engagement proportions for the markers of physical activity

\begin{tabular}{|c|c|c|c|c|c|c|c|c|c|c|}
\hline & Age & Walk/hike & Jog & Run & Bicycle & Aerobic & Swim & Tennis & Low intensity & FOSC \\
\hline Age & 1.00 & & & & & & & & & \\
\hline Walk/hike & -0.01 & & & & & & & & & \\
\hline Jog & $-0.16^{* *}$ & 0.09 & & & & & & & & \\
\hline Run & $-0.26^{* *}$ & $0.14^{*}$ & $0.41^{* *}$ & & & & & & & \\
\hline Bicycle & 0.07 & 0.08 & 0.05 & 0.04 & & & & & & \\
\hline Aerobic & $-0.11^{*}$ & $0.13^{*}$ & $0.28^{* *}$ & $0.17^{* *}$ & 0.06 & & & & & \\
\hline Swim & 0.03 & 0.01 & 0.04 & -0.04 & -0.03 & -0.06 & & & & \\
\hline Tennis & -0.01 & -0.08 & 0.07 & 0.04 & 0.01 & -0.04 & 0 & & & \\
\hline Low intensity & 0.00 & $0.21^{* *}$ & $0.12^{*}$ & $0.17^{* *}$ & $0.16^{* *}$ & $0.36^{* *}$ & -0.08 & -0.01 & & \\
\hline FOSC & 0.00 & $0.22^{* *}$ & -0.03 & -0.01 & $0.15^{* *}$ & 0.06 & 0.02 & 0.08 & -0.01 & \\
\hline \multicolumn{11}{|l|}{ Engagement } \\
\hline Young $(<52)$ & & 0.98 & 0.39 & 0.39 & 0.23 & 0.54 & 0.07 & 0.07 & 0.63 & 0.93 \\
\hline Old $(>52)$ & & 0.98 & 0.15 & 0.07 & 0.21 & 0.42 & 0.1 & 0.05 & 0.53 & 0.88 \\
\hline
\end{tabular}

${ }^{*} p<0.05,{ }^{* *} p<0.01$.

Key: FOSC, flights of stairs climbed.

structure of the brain in a younger state may have profound effects on delaying the onset of age-related neurodegenerative diseases.

Education and FOSC are associated with larger brain volume than predicted by CA supporting the idea of brain maintenance (Nyberg et al., 2012). Brain maintenance describes the preservation of brain measures (including cellular, neurochemical, volumetric, and systems-level activation patterns) in the face of advancing age and is argued to be a primary indicator of successful cognitive aging. Therefore, higher levels of education and FOSC daily are related to larger regional brain volumes, with respect to others with lower education and FOSC, thus suggesting that education and FOSC lead to the maintenance of brain volumes at a more youthful state.

It is important to stress that the current data are from a crosssectional study; therefore, causality cannot be tested. The theoretical framework underlying the present study is based on literature

Table 3

Regional relationships between the CA-BA difference and education and FOSC

\begin{tabular}{|c|c|c|c|c|c|}
\hline Measure & Region & Hemi. & $Z$ & $B$ & pTotal \\
\hline \multirow[t]{13}{*}{ Education } & Putamen & $\mathrm{L}$ & 2.10 & 0.045 & 7.6 \\
\hline & Putamen & $\mathrm{R}$ & 3.34 & 0.071 & 12.2 \\
\hline & Amygdala & $\mathrm{R}$ & 2.55 & 0.083 & 14.3 \\
\hline & Lateral orbitofrontal & $\mathrm{L}$ & 2.35 & 0.106 & 18.3 \\
\hline & Superior frontal & $\mathrm{R}$ & 2.39 & 0.035 & 6.1 \\
\hline & Frontal pole & $\mathrm{R}$ & 4.14 & 0.035 & 6.0 \\
\hline & Precuneus & $\mathrm{L}$ & 2.00 & 0.059 & 10.1 \\
\hline & Precentral & $\mathrm{R}$ & 3.26 & 0.026 & 4.5 \\
\hline & Superior temporal & $\mathrm{L}$ & 3.08 & 0.083 & 14.3 \\
\hline & Superior temporal & $\mathrm{R}$ & 2.66 & 0.075 & 12.9 \\
\hline & Middle temporal & $\mathrm{L}$ & 2.02 & 0.059 & 10.1 \\
\hline & Supramarginal & $\mathrm{L}$ & 2.27 & 0.041 & 7.0 \\
\hline & Lateral occipital & $\mathrm{R}$ & 2.30 & 0.082 & 14.1 \\
\hline \multirow[t]{13}{*}{ FOSC } & Amygdala & $\mathrm{R}$ & 2.35 & 0.120 & 12.4 \\
\hline & Accumbens & $\mathrm{R}$ & 2.11 & 0.013 & 1.4 \\
\hline & Rostral middle frontal & $\mathrm{R}$ & 2.48 & 0.024 & 2.5 \\
\hline & Caudal anterior cingulate & $\mathrm{L}$ & 2.08 & 0.013 & 1.4 \\
\hline & Caudal anterior cingulate & $\mathrm{R}$ & 2.24 & 0.014 & 1.4 \\
\hline & Posterior cingulate & $\mathrm{R}$ & 2.03 & 0.013 & 1.3 \\
\hline & Rostral anterior cingulate & $\mathrm{R}$ & 2.29 & 0.012 & 1.2 \\
\hline & Precuneus & $\mathrm{L}$ & 2.07 & 0.040 & 4.2 \\
\hline & Paracentral & $\mathrm{R}$ & 2.97 & 0.020 & 2.1 \\
\hline & Inferior temporal & $\mathrm{R}$ & 2.13 & 0.024 & 2.5 \\
\hline & Middle temporal & $\mathrm{L}$ & 2.45 & 0.087 & 9.0 \\
\hline & Transverse temporal & $\mathrm{R}$ & 2.53 & 0.012 & 1.2 \\
\hline & Insula & $\mathrm{R}$ & 2.24 & 0.018 & 1.9 \\
\hline
\end{tabular}

$\mathrm{Z}$ is calculated using 1000 bootstrap resamples and bias-corrected, accelerated confidence intervals, beta is the effect with units of years, and pTotal is the percent of the total effect each region represents. The total education effect is 0.97 and for FOSC is 0.58 .

Key: CA-BA, chronological age and brain age; FOSC, flights of stairs climbed; Hemi., hemisphere. showing that certain lifetime exposures, such as education and physical activity, have a positive effect on the aging brain. The findings from the present study support this theory. However, it is also possible that larger brain volumes may predispose individuals to obtain greater levels of education and seek out physical activity. Longitudinal and intervention studies may address the causal nature of the current findings and tease apart the concepts of predisposition and maintenance. For instance, BA, calculated using measurements obtained early in life, compared with lifetime educational attainment and levels of physical activity, obtained later in life, could be used to address the question of predisposition. A natural experiment comparing BA measures at the start of adulthood to BA measures calculated after completion of educational attainment would be useful in understanding whether educational attainment altered BA. This would test the theory that education contributes to brain maintenance. Likewise, an intervention study over a shorter time scale, using a physical activity protocol, could identify whether or not the intervention altered the rate of BA decline, helping to further our understanding of brain maintenance.

The pattern of brain regions showing a relationship with lifetime exposures is similar to the pattern of brain regions demonstrating the most substantial age-related declines (Raz et al., 1997). This suggests that these brain regions, in particular the temporal cortex, are more sensitive to the effects of aging but also sensitive to the lifetime exposures which may counteract some of the aging effects. The temporal cortex had significantly large contributions to the education and FOSC-related increases in the CA-BA measure. A previous aerobic walking study with older adults showed that increased functional connectivity was found within the temporal gyri and was associated with increased levels of brain-derived neurotrophic factor (Voss et al., 2012). Atrophy within these regions has also been implicated as an early indicator of Alzheimer's disease (Convit et al., 2000). Exercise is noted to moderate the amount of age-related atrophy within the temporal gyri (Bugg and Head, 2011). The volume of these regions also mediates age-related cognitive performance on memory strategies (Kirchhoff et al., 2014). Furthermore, greater walking distance predicted greater amounts of gray matter volume in regions of the occipital, temporal, and frontal lobes (Erickson et al., 2010).

Identification of biomarkers of healthy normal aging is an important step in understanding neurodegenerative decline (Jack et al., 2010). Using biomarkers of healthy aging could provide a measurement scale to identify deviation from normal. Their use may be particularly informative with respect to intervention studies. A biomarker of healthy aging could also provide a metric for identifying the effects of intervention programs. 


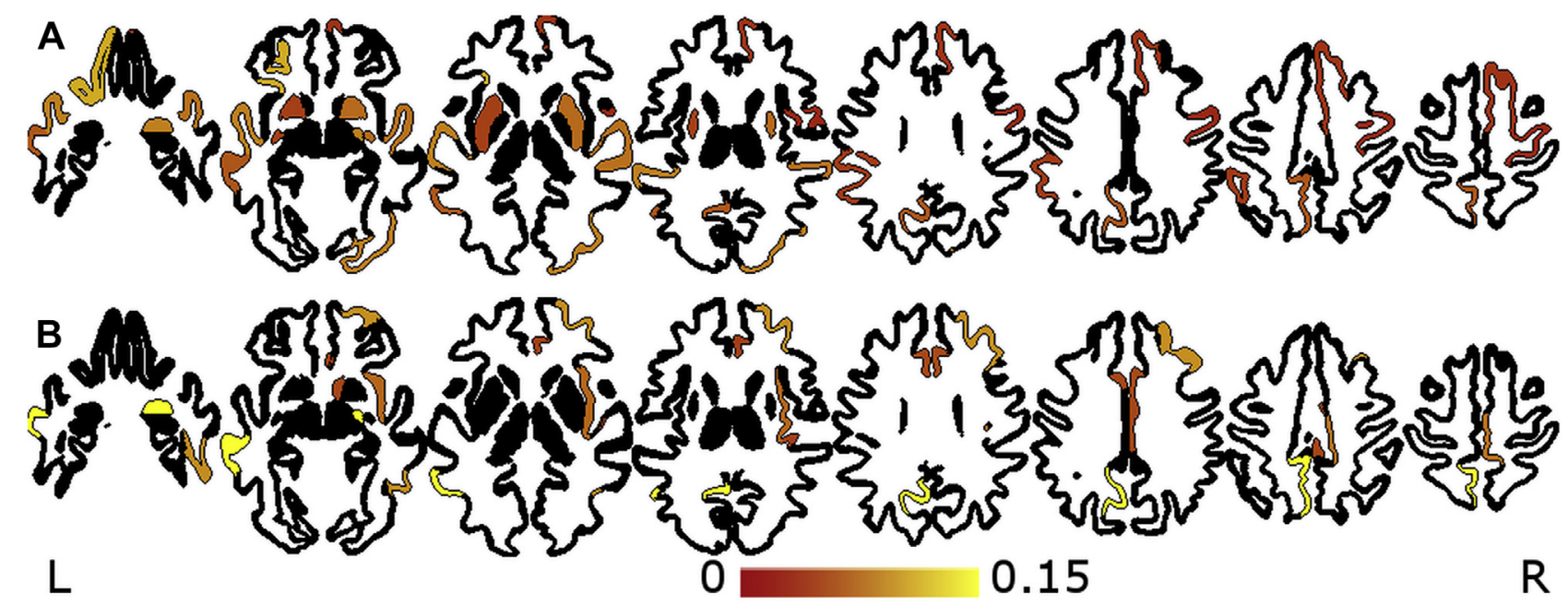

Fig. 2. Overlay of effect sizes for significant regions for education (A) and flights of stairs climbed (B).

Years of education and daily number of FOSC are both straightforward measures. The ease of collecting these measurements is to their advantage; however, they also each have drawbacks. Years of education does not assess quality of education (Manly et al., 2002). It also does not include age of acquisition of education. Although a large bolus of education is typically acquired early in life, the acquisition of higher education is not mandated to the young. Individuals that acquire 16 continuous years of education may differ from those that acquired their higher education later in life. Unfortunately, our measure does not make such distinctions.

The number of FOSC daily represents a proxy of a type of physical activity that has been used as a health-related fitness tool (Kennedy et al., 2007). These authors demonstrate that stair climbing enhances cardiovascular fitness. Stair climbing as an exercise also meets the minimum requirements for cardiorespiratory benefits and serves as a viable exercise, suitable for promoting physical activity (Teh and Aziz, 2002). In assessments of the validity of stair climbing, it was shown to not be significantly related to $\mathrm{VO}_{2}$ max assessed using a treadmill test in 138 participants (Resnicow et al., 2003). In another study of 76 post-menopausal, sedentary women using the Paffenbarger Physical Activity Questionnaire, sweat index was a better marker of measured kilocalories of energy expenditure than stair climbing (Laporte et al., 1983). The greatest support for the FOSC measure comes from the Harvard Alumni Health Study. This study demonstrated that in 13,485 participants, the number of FOSC and distance walked independently and significantly was related to decreased mortality rates by up to $25 \%$. Physical activity at light, moderate, or vigorous levels had no significant effects (Lee and Paffenbarger, 2000). Despite the validity of stair climbing as a physical activity, we measured it as a self-report which is inherently problematic (Prince et al., 2008). Despite this limitation, the other measures of physical activity were also selfreport and were not significantly related to our difference between CA and BA.

The assessment of FOSC is an item from the Paffenbarger Physical Activity Questionnaire (Paffenbarger et al., 1993). Overall, this is reliable and valid assessment of physical activity; however, there is limited evidence comparing the self-reported number of FOSC against measured flights climbed. Future research will need to validate self-reported stair climbing against actual measurements, when accuracy of pedometers increases for free-living conditions (De Cocker et al., 2012; Zhu and Lee, 2010).
Our results demonstrate that years of education and stair climbing, not other measures of physical activity, were related to the difference between CA and BA. This raises the question of what makes stair climbing different from other forms of physical activity in its relationship with brain health. One difference between stair climbing and other activities may be motivational. Many physical activities, for example, running or swimming, are done out of personal desire. Climbing a flight of stairs may also be motivated from personal desire, for example, for health reasons, and may be considered engagement in optional physical activity. On the other hand, climbing the stairs may be a physical activity engaged in out of necessity. In our cohorts, climbing stairs may reflect housing or transportation situations. The cohorts for this study were recruited from northern Manhattan, with a predominance of apartment buildings, and residential northern New Jersey, with a predominance of single family homes. Someone may live in a multilevel home and going upstairs and downstairs is simply a fact of daily life. Someone may also live in a multilevel apartment building, with no elevator, where again climbing flights of stairs is a fact of daily life. Public transportation in this region often involves ascending and descending flights of stairs to reach train platforms. A potentially complicating factor is that differences in housing may represent socioeconomic differences. In an observational study, pedestrians in higher socioeconomic regions had a significantly higher rate of optionally taking the stairs than pedestrians in lower socioeconomic regions (Ryan et al., 2011).

The nature of stair climbing also differs from many other forms of physical activity. Climbing stairs is often done repeatedly throughout the day, on most if not all days of the week, and represents small bouts of moderately intense physical activity. Engagement of the other activities we assessed typically occur less frequently throughout a typical week and for longer durations. Future work with measures of stair climbing will attempt to tease apart the motivation and socioeconomical differences behind taking the stairs. This will be useful in determining which aspect has the greatest impact on BA.

Stair climbing is also used as a marker of mobility. In a large sample of older adults, education was significantly related to stair climbing (Sainio et al., 2007). The authors discussed that the pathway of this effect appeared to be common chronic diseases, such as smoking and obesity. The lower educated participants had greater levels of these common diseases which limited their stair climbing ability. In our sample, there was no significant relationship 
between education and FOSC $(r=0.02)$, likely due to our eligibility screening.

Even with the limitations of the education and FOSC measures, they are both significantly related to the difference between chronological and biological age. Future work will improve the quantification of education and physical activity to more deeply probe the roles of these lifetime exposures, as well as exploring dosage effects of education and physical activity (Erickson et al., 2010).

Interestingly, engagement differs across the various physical activities. There are also correlations between the various measures and age. The more vigorous activities have lower numbers of participants engaging in them with advancing age. However, energy expenditure due to FOSC is unrelated to age. Engagement in FOSC, regardless of duration, is also unaffected by age. Therefore, FOSC is a physical activity that is engaged in by most adults. Current results suggest there may be a dose response with respect to estimated BA. Many people are already climbing the stairs at least once per day; our results suggest that climbing more flights of stairs per day may offer even greater benefit. This is encouraging because it demonstrates that FOSC has great potential as an intervention tool to promote brain health. There already exist many "Take the stairs" campaigns in office environments and public transportation centers. The current results demonstrate that these campaigns should also be expanded for older adults.

Prediction of CA using physiological measurements is gaining interest and is demonstrating its usefulness in the literature. Currently, there are multiple methods of deriving a BA estimate which are applicable for identifying differential rates of aging (Bunge and Whitaker, 2012; Franke et al., 2010; Wachinger et al., 2015), tracking recovery from traumatic brain injuries (Cole et al., 2015), and in measuring brain development in patients with multiple sclerosis (Aubert-Broche et al., 2014). When predicting CA, other methods have explained more variance in their data than in the methods used in this work (Cole et al., 2015; Franke et al., 2010; Wang et al., 2014). This is likely due to the voxel-vertex wise nature of their approaches using hundreds of thousands of brain measurements per participant. These techniques are quite powerful; however, they suffer from high sensitivity to accurate registrations between data and templates and processing steps. Franke et al. (2010) discuss and demonstrate the sensitivity of their measure to preprocessing choices (Franke et al., 2010).

The current approach used 84 brain measurements per participant using FreeSurfer extraction of regional volumes. This attempts to circumvent error due to preprocessing sensitivity using a robust extraction method that preserves the data in the original native collected space without excessive transformations. The current work also used the scaled subprofile modeling approach (Moeller et al., 1987). This method is straightforward and well established in the imaging field with demonstrated reproducibility (Moeller et al., 1999). Derived covariance patterns, such as those produced here, can serve as biomarkers of the healthy aging brain and may be forward applied to new data sets (Brickman et al., 2008). This is in common with the other described BA calculations.

Using 95\% of the variance in the brain data, this approach accounted for $47 \%$ of the variance in CA. Although other methods may account for larger amounts of variance in their data, our preference was to minimize sensitivity to processing choices and use established methods from the literature. It is also anticipated that the greatest increase in variance accounted for in CA will come from the combination of multiple physiological measurements.

\section{Conclusions}

Differences between chronological and BA were significantly related to years of education and the daily number of FOSC. These results support the idea that more education and climbing a greater number of stairs are healthy lifestyle habits as reflected by regional brain volumes being larger than expected by CA. The identified full brain pattern of BA also provides a biomarker which may be useful for predicting whether BA is consistent or inconsistent with CA.

\section{Disclosure statement}

All authors verify that they have no conflict of interest related to this work.

\section{Acknowledgements}

This research was supported by grants from the National Institute on Aging (AG035061, PI Dr Jason Steffener, AG044467, PI Dr Qolamreza Razlighi, and AG026158, AG038465, PI Dr Yaakov Stern). The content is solely the responsibility of the authors and does not necessarily represent the official views of the National Institutes of Health. The sponsors had no role in the study design, data collection, analysis or interpretation, writing of the report, or decision to submit the article for publication.

\section{References}

Ahlskog, J.E., Geda, Y.E., Graff-Radford, N.R., Petersen, R.C., 2011. Physical exercise as a preventive or disease-modifying treatment of dementia and brain aging. Mayo Clin. Proc. 86, 876-884

Ainsworth, B.E., Haskell, W.L., Whitt, M.C., Irwin, M.L., Swartz, A.M., Strath, S.J. O’Brien, W.L., Bassett Jr., D.R., Schmitz, K.H., Emplaincourt, P.O., Jacobs Jr., D.R., Leon, A.S., 2000. Compendium of physical activities: an update of activity codes and MET intensities. Med. Sci. Sports Exerc. 32, S498-S504.

American Psychiatric Association, 1994. Diagnostic and statistical manual of mental disorders. In: 4th ed. American Psychiatric Press, Washington, DC.

Aubert-Broche, B., Fonov, V., Narayanan, S., Arnold, D.L., Araujo, D., Fetco, D., Till, C. Sled, J.G., Banwell, B., Collins, D.L., Canadian Pediatric Demyelinating Disease Network, 2014. Onset of multiple sclerosis before adulthood leads to failure of age-expected brain growth. Neurology 83, 2140-2146.

Bassett, D.R., Vachon, J.A., Kirkland, A.O., Howley, E.T., Duncan, G.E., Johnson, K.R. 1997. Energy cost of stair climbing and descending on the college alumnus questionnaire. Med. Sci. Sports Exerc. 29, 1250-1254.

Brickman, A.M., Habeck, C., Ramos, M.A., Scarmeas, N., Stern, Y., 2008. A forward application of age associated gray and white matter networks. Hum. Brain Mapp. 29, 1139-1146.

Bugg, J.M., Head, D., 2011. Exercise moderates age-related atrophy of the medial temporal lobe. Neurobiol. Aging 32, 506-514.

Bunge, S.A., Whitaker, K.J., 2012. Brain imaging: your brain scan doesn't lie about your age. Curr. Biol. 22, R800-R801.

Chao, A., Connell, C.J., Jacobs, E.J., McCullough, M.L., Patel, A.V., Calle, E.E. Cokkinides, V.E., Thun, M.J., 2004. Amount, type, and timing of recreational physical activity in relation to colon and rectal cancer in older adults: the Cancer Prevention Study II Nutrition Cohort. Cancer Epidemiol. Biomarkers Prev. 13, 2187-2195.

Cole, J.H., Leech, R., Sharp, D.J., For the Alzheimer's Disease Neuroimaging Initiative, 2015. Prediction of brain age suggests accelerated atrophy after traumatic brain injury. Ann. Neurol. 77, 571-581.

Convit, A., de Asis, J., de Leon, M.J., Tarshish, C.Y., De Santi, S., Rusinek, H., 2000. Atrophy of the medial occipitotemporal, inferior, and middle temporal gyri in non-demented elderly predict decline to Alzheimer's disease. Neurobiol. Aging $21,19-26$.

Dale, A.M., Fischl, B., Sereno, M.I., 1999. Cortical surface-based analysis. I. Segmentation and surface reconstruction. Neuroimage 9, 179-194.

De Cocker, K.A., De Meyer, J., De Bourdeaudhuij, I.M., Cardon, G.M., 2012. Nontraditional wearing positions of pedometers: validity and reliability of the Omron HJ-203-ED pedometer under controlled and free-living conditions. J. Sci. Med. Sport 15, 418-424.

Erickson, K.I., Raji, C.A., Lopez, O.L., Becker, J.T., Rosano, C., Newman, A.B., Gach, H.M., Thompson, P.M., Ho, A.J., Kuller, L.H., 2010. Physical activity predicts gray matter volume in late adulthood: the Cardiovascular Health Study. Neurology 75, $1415-1422$

Fischl, B., 2012. FreeSurfer. Neuroimage 62, 774-781.

Fischl, B., Salat, D.H., Busa, E., Albert, M., Dieterich, M., Haselgrove, C., van der Kouwe, A., Killiany, R., Kennedy, D., Klaveness, S., Montillo, A., Makris, N. Rosen, B., Dale, A.M., 2002. Whole brain segmentation: automated labeling of neuroanatomical structures in the human brain. Neuron 33, 341-355.

Fischl, B., van der Kouwe, A., Destrieux, C., Halgren, E., Ségonne, F., Salat, D.H., Busa, E., Seidman, L.J., Goldstein, J., Kennedy, D., Caviness, V., Makris, N., 
Rosen, B., Dale, A.M., 2004. Automatically parcellating the human cerebral cortex. Cereb. Cortex 14, 11-22.

Franke, K. Ziegler, G., Klöppel, S, Gaser, C. Alzheimer's Disease Neuroimaging Initiative, 2010. Estimating the age of healthy subjects from T1-weighted MRI scans using kernel methods: exploring the influence of various parameters. Neuroimage 50, 883-892.

Habeck, C., Krakauer, J.W., Ghez, C., Sackeim, H.A., Eidelberg, D., Stern, Y., Moeller, J.R., 2005. A new approach to spatial covariance modeling of functional brain imaging data: ordinal trend analysis. Neural Comput. 17, 1602-1645.

Habeck, C., Stern, Y., 2007. Neural network approaches and their reproducibility in the study of verbal working memory and Alzheimer's disease. Clin. Neurosci. Res. 6, 381-390.

Irimia, A., Torgerson, C.M., Goh, S.-Y.M., Van Horn, J.D., 2014. Statistical estimation of physiological brain age as a descriptor of senescence rate during adulthood. Brain Imaging Behav. 9, 678-689.

Jack Jr., C.R., Knopman, D.S., Jagust, W.J., Shaw, L.M., Aisen, P.S., Weiner, M.W., Petersen, R.C., Trojanowski, J.Q., 2010. Hypothetical model of dynamic biomarkers of the Alzheimer's pathological cascade. Lancet Neurol. 9, 119-128.

Kennedy, R.A., Boreham, C.A.G., Murphy, M.H., Young, I.S., Mutrie, N., 2007. Evaluating the effects of a low volume stairclimbing programme on measures of health-related fitness in sedentary office workers. J. Sports Sci. Med. 6, $448-454$.

Kirchhoff, B.A., Gordon, B.A., Head, D., 2014. Prefrontal gray matter volume mediates age effects on memory strategies. Neuroimage 90, 326-334.

Laporte, R.E., Black-sandler, R., Cauley, J.A., Link, M., Bayles, C., Marks, B., 1983. The assessment of physical activity in older women: analysis of the interrelationship and reliability of activity monitoring, activity surveys, and caloric intake. J. Gerontol. 38, 394-397.

Lee, I.-M., Paffenbarger, R.S., 2000. Associations of light, moderate, and vigorous intensity physical activity with longevity: the Harvard Alumni Health Study, Am. J. Epidemiol. 151, 293-299.

Manly, J.J., Jacobs, D.M., Touradji, P., Small, S.A., Stern, Y., 2002. Reading level attenuates differences in neuropsychological test performance between African American and white elders. J. Int. Neuropsychol. Soc. 8, 341-348.

Mattis, S., 1988. Dementia rating scale. Psychological assessment resources, Odessa, FL.

Moeller, J.R., Nakamura, T., Mentis, M.J., Dhawan, V., Spetsieres, P., Antonini, A., Missimer, J., Leenders, K.L., Eidelberg, D., 1999. Reproducibility of regional metabolic covariance patterns: comparison of four populations. J. Nucl. Med. 40, 1264-1269.

Moeller, J.R., Strother, S.C., Sidtis, J.J., Rottenberg, D.A., 1987. Scaled subprofile model: a statistical approach to the analysis of functional patterns in positron emission tomographic data. J. Cereb. Blood Flow Metab. 7, 649-658.

Nithianantharajah, J., Hannan, A.J., 2009. The neurobiology of brain and cognitive reserve: mental and physical activity as modulators of brain disorders. Prog. Neurobiol. 89, 369-382.

Nyberg, L., Lövdén, M., Riklund, K., Lindenberger, U., Bäckman, L., 2012. Memory aging and brain maintenance. Trends Cogn. Sci. 16, 292-305.
Paffenbarger Jr., R.S., Blair, S.N., Lee, I.-M., Hyde, R.T., 1993. Measurement of physical activity to assess health effects in free-living populations. Med. Sci. Sports Exerc $25,60-70$.

Prince, S.A., Adamo, K.B., Hamel, M.E., Hardt, J., Connor Gorber, S., Tremblay, M. 2008. A comparison of direct versus self-report measures for assessing physical activity in adults: a systematic review. Int. J. Behav. Nutr. Phys. Act 5, 56.

Raz, N., Ghisletta, P., Rodrigue, K.M., Kennedy, K.M., Lindenberger, U., 2010. Trajectories of brain aging in middle-aged and older adults: regional and individual differences. Neuroimage 51, 501-511.

Raz, N., Gunning, F.M., Head, D., Dupuis, J.H., McQuain, J., Briggs, S.D., Loken, W.J. Thornton, A.E., Acker, J.D., 1997. Selective aging of the human cerebral cortex observed in vivo: differential vulnerability of the prefrontal gray matter. Cereb. Cortex 7, 268-282.

Resnicow, K., McCarty, F., Blissett, D., Wang, T., Heitzler, C., Lee, R.E., 2003. Validity of a modified CHAMPS physical activity questionnaire among African-Americans. Med. Sci. Sports Exerc. 35, 1537-1545.

Ryan, J., Lyon, K., Webb, O.J., Eves, F.F., Ryan, C.G., 2011. Promoting physical activity in a low socioeconomic area: results from an intervention targeting stair climbing. Prev. Med. 52, 352-354.

Sainio, P., Martelin, T., Koskinen, S., Heliövaara, M., 2007. Educational differences in mobility: the contribution of physical workload, obesity, smoking and chronic conditions. J. Epidemiol. Community Health 61, 401-408.

Sowell, E.R., Thompson, P.M., Toga, A.W., 2004. Mapping changes in the human cortex throughout the span of life. Neuroscientist 10, 372-392.

Spetsieris, P.G., Eidelberg, D., 2010. Scaled subprofile modeling of resting state imaging data in Parkinson's disease: methodological issues. Neuroimage 54, 2899-2914.

Teh, K.C., Aziz, A.R., 2002. Heart rate, oxygen uptake, and energy cost of ascending and descending the stairs. Med. Sci. Sports Exerc. 34, 695-699.

Terry, R.D., DeTeresa, R., Hansen, L.A., 1987. Neocortical cell counts in normal human adult aging. Ann. Neurol. 21, 530-539.

Thacker, E.L., Chen, H., Patel, A.V., McCullough, M.L., Calle, E.E., Thun, M.J. Schwarzschild, M.A., Ascherio, A., 2008. Recreational physical activity and risk of Parkinson's disease. Mov. Disord. 23, 69-74.

Voss, M.W., Erickson, K.I., Prakash, R.S., Chaddock, L., Kim, J.S., Alves, H., Szabo, A., Phillips, S.M., Wójcicki, T.R., Mailey, E.L., Olson, E.A., Gothe, N., Vieira-Potter, V.J. Martin, S.A., Pence, B.D., Cook, M.D., Woods, J.A., McAuley, E., Kramer, A.F., 2012 Neurobiological markers of exercise-related brain plasticity in older adults. Brain Behav. Immun. 28, 90-99.

Wachinger, C., Golland, P., Kremen, W., Fischl, B., Reuter, M., Alzheimer's Disease Neuroimaging Initiative, 2015. BrainPrint: a discriminative characterization of brain morphology. Neuroimage 109, 232-248.

Wang, J., Li, W., Miao, W., Dai, D., Hua, J., He, H., 2014. Age estimation using cortica surface pattern combining thickness with curvatures. Med. Biol. Eng. Comput. $52,331-341$.

Zhu, W., Lee, M., 2010. Invariance of wearing location of Omron-BI pedometers: a validation study. J. Phys. Act. Health 7, 706-717. 